\title{
QUELQUES CONSÉQUENCES DE L'INTRODUCTION DE DEUX ESPECES DE MACROPHYTES, ELODEA CANADENSIS MICHAUX ET ELODEA NUTTALLII ST. JOHN, DANS LES ÉCOSYSTËMES AQUATIQUES CONTINENTAUX : EXEMPLE DE LA PLAINE D'ALSACE ET DES VOSGES DU NORD (NORD-EST DE LA FRANCE).
}

G. THIEBAUT (1), T. ROLLAND (2), F. ROBACH (2),

M. TREMOLIERES (2), S. MULLER (1).

(1) Centre de Recherches Ecologiques de l'Université de Metz, Equipe de Phytoécologie, lle du Saulcy, 57045 Metz, France.

(2) Université Louis Pasteur, Institut de Botanique, Laboratoire de Botanique et d'Ecologie végétale, CEREG URA 95 CNRS, 28 rue Goethe, 67083 Strasbourg, France.

\section{RÉSUMÉ}

L'introduction au XIXème et XXème siècle, de deux espèces d'élodées Elodea canadensis Michaux et Elodea nuttallii St. John, dans les cours d'eau du nord-est de la France, a induit des modifications dans la composition floristique et le fonctionnement des écosystèmes aquatiques. L'analyse comparative de la distribution géographique de ces deux hydrophytes dans les eaux calcaires (plaine d'Alsace) et acides (Vosges du Nord) a permis de préciser leur écologie. Après des phases successives d'expansion des deux espèces, celles-ci se sont intégrées dans les phytocénoses aquatiques. L'introduction dans les écosystèmes aquatiques d'Elodea canadensis et d'E. nuttallii a induit des phénomènes de compétition interspécifique, en faveur essentiellement d'Elodea nuttallii. Cette dernière apparaît comme l'espèce la plus compétitive dans les cours d'eau eutrophes de la plaine d'Alsace (aptitude à accumuler le phosphore, sensibilité moindre à l'azote ammoniacal). Les élodées peuvent réaliser une épuration biologique, mais provoquent, lors de leur décomposition, une eutrophisation secondaire des eaux en absence de faucardage.

SOME CONSEQUENCES OF THE INTRODUCTION OF TWO MACROPHYTE SPECIES, ELODEA CANADENSIS MICHAUX AND ELODEA NUTTALLII ST. JOHN, IN CONTINENTAL AQUATIC ECOSYSTEMS : EXAMPLE OF TWO AREAS IN THE NORTH-EAST OF FRANCE : ALSACE PLAIN AND NORTHERN VOSGES.

\begin{abstract}
The introduction of two species of elodeids, Elodea canadensis Michaux and Elodea nuttallii St. John during the $X I X$ th and $X X$ th centuries had a significant effect on the aquatic ecosystems in the North-East of France. These species invaded the streams preventing the full development of the native species. Their distribution is determined by ecological factors (mineralization, nutrient loads) in the calcareous streams of the Alsace Plain and in the soft waters of the Northern Vosges. They are able to grow in the same area and the same trophic range, but Elodea nuttallii seems to be less sensitive to ammonium and much more competitive than E. canadensis in the Alsace plain. In this area, it was demonstrated that Elodea nuttallii,
\end{abstract}


which is widely distributed especially in eutrophic waters, also exhibits the highest phosphate uptake and accumulation capacity. This is why Elodea nuttallii increasingly replaced $E$. canadensis in streams. These aquatic macrophytes improve water quality. But, if not harvested, they cause stream eutrophication after their decomposition.

\section{INTRODUCTION}

Les milieux aquatiques lotiques se comportent comme des systèmes écologiques particulièrement fragiles (CARBIENER et al., 1990). L'introduction dans le nord-est de la France, de deux espèces de macrophytes aquatiques Elodea canadensis Michaux et Elodea nuttallii St. John et leur intégration dans les phytocénoses aquatiques ne sont pas sans effet sur la composition spécifique des communautés végétales autochtones et sur la qualité des eaux. Aussi, l'étude de la distribution et de l'écologie des deux élodées dans les cours d'eau du nordest de la France apparaît comme un élément de la compréhension des mécanismes en jeu dans les processus d'intégration et de développement des deux espèces.

Le bilan de la répartition actuelle des deux espèces d'élodées dans les Vosges du Nord et en plaine d'Alsace a donc été dressé, afin de juger de leur potentialité à se développer dans ce territoire. Leur écologie est précisée et les facteurs responsables de leur expansion dans les ruisseaux sont recherchés. Enfin, les conséquences écologiques de l'introduction des deux espèces d'élodées dans les cours d'eau sont analysées.

\section{MÉTHODES ET SITES D'ÉTUDES}

\section{Présentation des deux secteurs d'études}

Ils sont situés dans le nord-est de la France et sont soumis à un climat de type subcontinental.

\section{Les Vosges du Nord}

Le réseau hydrographique draine un substrat gréseux (grès vosgien, grès bigarré, grès d'Annweiler) couvert de forêts de feuillus et de résineux ou de zones tourbeuses. Les cours d'eau sont faiblement minéralisés $(\mathrm{pH} 5,4$ à 7,2 ; conductivité 60 à $110 \mu \mathrm{S} / \mathrm{cm})$, oligotrophes à hypertrophes à l'aval du secteur (THIEBAUT et MULLER, 1995). La température moyenne annuelle est de $8,6^{\circ} \mathrm{C}$ et des précipitations moyennes annuelles atteignent $900 \mathrm{~mm}$ à Mouterhouse.

\section{La plaine d'Alsace}

Le réseau hydrographique de la plaine alluviale ello-rhénane draine un substrat bicarbonaté calcaire présentant une relative homogénéité vis-à-vis de la minéralisation $(\mathrm{pH} 7,5$ à 8,0 ; conductivité 500 à $1000 \mu \mathrm{S} / \mathrm{cm}$ ). Les cours d'eau, situés essentiellement en milieu prairial, sont des eaux de surface et des rivières phréatiques, principalement alimentées par des résurgences de la nappe phréatique des graviers glaciaires du Rhin. Les sources, issues directement de la nappe, possèdent des caractéristiques physico-chimiques relativement constantes. Les eaux sont limpides, pures, fraîches $\left(9-13^{\circ} \mathrm{C}\right)$, bicarbonatées calciques, oligosaprobes et oligotrophes aux sources (CARBIENER et al., 1990). La température moyenne annuelle de la zone d'étude est de $9,7^{\circ} \mathrm{C}$ et les précipitations moyennes annuelles sont comprises entre 600 et $700 \mathrm{~mm}$. Le climat local est fortement influencé par la forêt ello-rhénane et le fleuve, se traduisant par une hausse de l'hygrométrie et une atténuation des extrêmes thermiques.

\section{Méthodes}

\section{Le choix des stations d'études}

Dans les Vosges du Nord, sur 18 cours d'eau prospectés, 14 stations (8 stations à Elodea canadensis et 6 sites à $E$. nuttallii) ont été retenues, de manière à obtenir une échelle de trophie allant de l'amont oligo-mésotrophe à l'aval hypertrophe (THIEBAUT et MULLER, 1995). 
Pour le réseau alsacien, 30 cours d'eau représentatifs ont été également choisis, de manière à couvrir une gamme trophique étendue. Dans les ruisseaux alsaciens, Elodea canadensis se développe dans 15 stations, alors que $E$. nuttallii est présent sur 20 stations.

\section{Etude floristique}

Des relevés de la végétation aquatique ont été effectués en utilisant la méthode phytosociologique (coefficient d'Abondance-Dominance de BRAUN-BLANQUET, 1964) sur les deux réseaux hydrographiques. La détermination des végétaux a été effectuée jusqu'à l'espèce à l'aide de la "Nouvelle Flore de Belgique, du Grand-Duché du Luxembourg, du nord de la France et des régions voisines "(LAMBINON et al., 1993).

Des tableaux phytosociologiques ont été élaborés à partir de la fréquence et en fonction des paramètres physico-chimiques (Tableaux I et II).

\section{Physico-chimie des cours d'eau}

De 1993 à 1995, des échantillons d'eau ont été prélevés dans ces stations chaque trimestre dans les Vosges du Nord et mensuellement en plaine d'Alsace. $\mathrm{Le} \mathrm{pH}$, la conductivité, l'alcalinité, l'azote nitrique $\mathrm{N}-\mathrm{NO}_{3}{ }^{-}$, l'azote ammoniacal $\mathrm{N}-\mathrm{NH}_{4}{ }^{+}$et les orthophosphates $\mathrm{P}_{-} \mathrm{PO}_{4}{ }^{3-}$ sont analysés selon les normes AFNOR en vigueur.

\section{RÉSULTATS}

\section{Description et historique de l'introduction des deux espèces}

\section{Description morphologique des deux macrophytes}

Elodea canadensis Michaux est une plante submerse, vert sombre, dont la tige grêle porte des feuilles sessiles et ovales verticillées par trois. L'espèce est dioïque, mais seules les fleurs femelles sont rencontrées en Europe. La multiplication est donc exclusivement végétative. La floraison, lorsqu'elle se produit, a lieu de juillet à août. Elodea nuttallii St. John a également un développement submerse, avec une tige dressée fine qui se distingue d'Elodea canadensis par ses feuilles plus étroites, courbées, crispées et à terminaisons en pointes aiguës, ainsi que par ses entre-noeuds plus longs et par sa coloration vert pâle. L'espèce est dioïque et les fleurs mâles et femelles sont rencontrées en Europe ; la floraison a lieu de juillet à août (SELL, 1968).

\section{Historique de l'apparition des deux espèces}

Originaire d'Amérique du nord, Elodea canadensis a été introduite en Europe à partir de I'Jrlande en 1836, trouvée en Angleterre en 1842 puis en France en 1845. Cette espèce est signalée pour la première fois en Lorraine en 1877 par GODRON (1877) et en Alsace en 1878 (SOCIÉTÉ D'ETUDE DE LA FLORE D'ALSACE, 1982). Elle a dû apparaître dans les Vosges du Nord à la fin du XIXème ou au début du XXème siècle (aucune donnée précise n'existe) et a progressivement colonisé la plupart des réseaux hydrographiques. Après un envahissement important au départ dans toute l'Europe tempérée, l'espèce a régressé suite à des mécanismes de compétition. La phase actuelle est celle de l'intégration d'Elodea canadensis dans les phytocénoses aquatiques (MERIAUX, 1979).

Egalement originaire d'Amérique du Nord, Elodea nuttallii a été récoltée pour la première fois en Belgique en 1939 (LAMBINON et al., 1993), puis en 1941 aux Pays-Bas et en Allemagne. En France, cette espèce a été observée d'abord en Alsace, dans les années 1950 (SELL, 1959 ; GEISSERT et al., 1985). Sa présence a été ensuite notée dans le département de la Meuse (DE LANGHE et D'HOSE, 1974), puis dans celui des Ardennes, enfin dans ceux du Nord (MÉRIAUX et GEHU, 1979) et du Pas-de-Calais (MÉRIAUX, 1977). Dans les Vosges du Nord, la première observation est due à P. WOLFF (in ENGEL et al., 1979). Actuellement, il se produit un envahissement des stations avec régression des autres espèces. Mais Elodea nuttallii peut, après une phase de forte extension, s'intégrer dans la végétation aquatique. En Europe, cette 
espèce se développe principalement dans les eaux eutrophes, riches en calcaire, moyennement polluées (WEBER-OLDECOP, 1977).

Aire de répartition d'Elodea nuttallii St. John et d'Elodea canadensis Michaux dans le nord-est de la France

Distribution des 2 élodées dans les groupements de macrophytes des Vosges du Nord

Une séquence de bioindication de l'eutrophisation par les macrophytes aquatiques a été établie dans les cours d'eau faiblement minéralisés des Vosges du Nord (MULLER, 1990; THIEBAUT et MULLER, 1995). Elle est constituée de quatre groupements végétaux notés de " $A$ » ̀̀ « $D$ » (tableau $)$ :

\section{Tableau I}

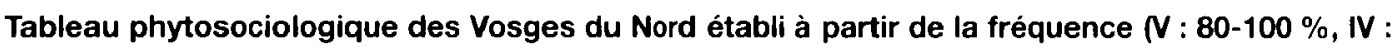
60-80 \%, III : 40-60\%, II : 20-40\%, I : 0-20\%) et en fonction des paramètres physico-chimiques (moyenne et écart-type).

Le coefficient moyen d'Abondance-Dominance est indiqué entre parenthèses.

\section{Table I}

Phytosociological table based on frequence of occurrence of species in community of Northern Vosges (V : 80-100\%, IV : 60-80\%, III : 40-60\%, II : 20-40\%, I : 0-20\%) and physico-chemical variables correlated (Means and standard deviation calculated on one year).

In the brackets, the mean Abundance-Dominance coefficient is indicated.

\begin{tabular}{|c|c|c|c|c|}
\hline 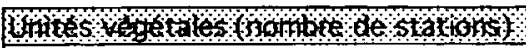 & A (3) & $6(10)$ & (110) & $0(6)$ \\
\hline Potamogeton polygonifolius & $V(2)$ & $V(1)$ & & . \\
\hline Cardamine amara & I(1) & $\|(1)$ & & \\
\hline Glyceria fluitans & $\operatorname{IV}(2)$ & $V(1)$ & $V(+)$ & $V(+)$ \\
\hline Fontinalis antipyretica & I(1) & $\|(1)$ & $\|(1)$ & III (2) \\
\hline Mentha aquatica & $1(+)$ & I(1) & $\|(+)$ & $\|(+)$ \\
\hline Sparganium erectum & IV (1) & III (1) & $1(+)$ & $1(+)$ \\
\hline Veronica beccabunga & I(1) & & III $(+)$ & $1(+)$ \\
\hline Sparganium emersum & $1(+)$ & $\mathrm{III}(1)$ & $\mathrm{IV}(1)$ & $\operatorname{III}(1)$ \\
\hline Callitiche hamulata & & HII(1) & $V(1)$ & $V(2)$ \\
\hline Callitriche platycarpa & . & $\operatorname{III}(2)$ & $\operatorname{III}(2)$ & $\|(1)$ \\
\hline Callitriche stagnalis & & $\operatorname{IV}(t)$ & $\mathrm{IV}(+)$ & $\| I(+)$ \\
\hline Lemna minor & . & $\|(+)$ & III (+) & IV $(+)$ \\
\hline Ranunculus peltatus & . & $\|(1)$ & $\|(2)$ & $\|(3)$ \\
\hline Berula erecta & . & $\|(1)$ & $\mathrm{I}(1)$ & $1(2)$ \\
\hline Potamogeton crispus & & 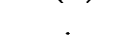 & (1) & \\
\hline Myriophyllum alterniflorum & . & . & $\|(+)$ & \\
\hline Potamogeon alpinus & . & . & $1(+)$ & . \\
\hline Potamogeton variffolius & & . & $\mathrm{I}(+)$ & \\
\hline Phalaris arundinacea & & & $V(1)$ & $V(+)$ \\
\hline Glyceria maxima & . & . & III (+) & $\|(+)$ \\
\hline Nasturtium officinale & . & . & III (1) & III (+) \\
\hline Potamogeton berchtoldii & . & . & $1(2)$ & $1(+)$ \\
\hline & . & & $1(2)$ & $1(+)$ \\
\hline \multirow{2}{*}{ 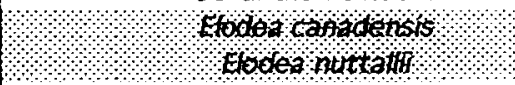 } & 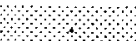 & \multirow{2}{*}{$8+$} & the & $41)$ \\
\hline & & & $(2)$ & $\sin (3)$ \\
\hline $\begin{array}{l}\text { Callitriche obtusangula } \\
\text { Myosotis scompioides }\end{array}$ & . & . & 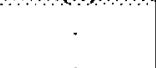 & $V(3)$ \\
\hline $\mathrm{pH}$ & $6,2(0,5)$ & $6,4(0,3)$ & $6,9(0,2)$ & $7,0(0,4)$ \\
\hline conductivité $(\mu \mathrm{S} / \mathrm{cm})$ & $59(13)$ & $50(9)$ & $77(23)$ & $82(25)$ \\
\hline $\mathrm{N}-\mathrm{NH}_{4}{ }^{+}(\mu \mathrm{g} / \mathrm{I})$ & $51(97)$ & $88(164)$ & $138(170)$ & $157(136)$ \\
\hline $\mathrm{P}^{-\mathrm{PO}_{4} 3^{-}}(\mu \mathrm{g} / \mathrm{l})$ & $30(40)$ & $31(40)$ & $120(176)$ & $132(65)$ \\
\hline $\mathrm{N}-\mathrm{NO}_{3}{ }^{-}(\mathrm{mg} / \mathrm{l})$ & $0,5(0,4)$ & $0,4(0,3)$ & $0,6(0,5)$ & $0,7(0,3)$ \\
\hline
\end{tabular}


- le groupement oligotrophe "A " est caractérisé par Potamogeton polygonifolius Pourr. Dans les zones de source à faible circulation d'eau, le potamot peut y être associé à des sphaignes, dans les eaux courantes sur substrat rocheux à Scapania undulata (L.) Dum.

- le groupement oligo-mésotrophe “ B " est caractérisé par l'apparition de Callitriche hamulata Kütz. ex-Koch, C. platycarpa Kütz, C. stagnalis Scop., accompagnés de Ranunculus peltatus Schrank et Sparganium emersum Rehm, qui s'y développent en compagnie de Potamogeton polygonifolius avec, en outre, dans les faciès lénitiques, Lemna minor $L$.

- le groupement mésotrophe “ $C$ » se distingue des deux unités précédentes par la disparition de Potamogeton polygonifolius et l'apparition, avec le cortège de "B ", d'Elodea canadensis, E. nuttallii, Potamogeton berchtoldii Fieb. II s'y ajoute quelques espèces rares Potamogeton alpinus Balb., P. x. variifolius Thore, Myriophyllum alterniflorum DC. et Oenanthe fluviatilis (Bab.) Colem.

- le groupement eutrophe “ D " s'individualise par l'apparition de Callitriche obtusangula Le Gall, ainsi que la plus grande fréquence d'Elodea nuttallii et de Nasturtium officinale R. Brown. Les algues du genre Vaucheria, Oedogonium, Binuclearia peuvent y former des peuplements importants en été.

Dans les eaux courantes des Vosges du Nord, les deux espèces d'élodées se développent habituellement en compagnie de Callitriche hamulata, C. platycarpa, Ranunculus peltatus, ainsi que d'espèces plus rares dans ce territoire comme Myriophyllum alterniflorum, Oenanthe fluviatilis, Potamogeton alpinus et $P$. variifolius. Des différences apparaissent, toutefois, dans la distribution géographique de ces deux espèces. Ainsi, Elodea canadensis apparait sur une seule station en compagnie de Potamogeton polygonifolius (correspondant au type “ $B$ " de la séquence d'eutrophisation, MULLER, 1990). Plus fréquemment, cette espèce se trouve dans le groupement " C ", alors qu'Elodea nuttallii semble rencontrer son optimum dans le type " D " à Callitriche obtusangula (Tableau I). Les deux espèces d'élodées Elodea canadensis et $E$. nuttallii n'ont été trouvées ensemble que sur deux stations.

Distribution des deux élodées dans les groupements de macrophytes de la plaine d'Alsace

CARBIENER et ORTSCHEIT (1987) ont établi, sur les phytocénoses des rivières phréatiques du Ried alsacien, un système de bioindication de l'eutrophisation en milieu minéralisé (" $A, B, C, D$ et $E$ "), déterminé par les phosphates et l'azote ammoniacal, allant de l'oligotrophe " $A$ » au plus eutrophe « $E$ ". Par la suite, CARBIENER et al. (1990), ROBACH et al. (1991) ont étendu cette échelle de bioindication par les macrophytes aux rivières eutrophes et hypertrophes de la plaine d'Alsace. Cette nouvelle séquence est constituée de 6 échelons notés de « $A$ " ̀̀ " $F$ » (Tableau II) :

- la communauté végétale " $A$ " oligotrophe se caractérise par la présence de Potamogeton coloratus Hornem., Batrachospermum monoliforme, Juncus subnodulus Schrank et Chara hispida L.

- la communauté végétale " B " oligo-mésotrophe est dominée par Berula erecta Huds., Callitriche obtusangula y est très faiblement représentée. Elle est surtout caractérisée par l'absence de Potamogeton coloratus.

- la communauté végétale “ $C$ » méso-eutrophe est caractérisée par le développement important de Callitriche obtusangula et par l'apparition de Lemna trisulca L., Fontinalis antipyretica Hedw., Potamogeton friesii Rupr., Elodea canadensis.

- la communauté végétale " $D$ " rhéophile et eutrophe est caractérisée par Potamogeton crispus L., Zannichellia palustris L., Groenlandia densa (L.) Fourr. et Ranunculus trichophyllus Chaix (plante rarement présente, mais typique du groupement). Nasturtium officinale et Lemna trisulca $L$. sont très représentatives de cette communauté. 
- la communauté végétale " $E$ " eutrophe se caractérise par Potamogeton pectinatus L., Elodea nuttallii, Myriophyllum spicatum L. et Potamogeton perfoliatus L. Oenanthe fluviatilis y est présent. Nasturtum officinale régresse et Lemna trisulca se maintient.

- la communauté végétale " $F$ " correspond aux zones les plus eutrophes. Elle est caractérisée par la présence de végétaux du type " $E$ " auxquels s'adjoignent obligatoirement soit Potamogeton nodosus Poiret, soit Potamogeton lucens L. qui s'excluent mutuellement dans le secteur central de la plaine d'Alsace. Ranunculus fluitans Lam. y trouve son optimum de développement.

\section{Tableau ||}

Tableau phytosociologique de la plaine d'Alsace établi à partir de la fréquence $(\mathrm{V}: 80-100 \%$, IV : 60$80 \%$, III : 40-60\%, II : $20-40 \%, I: 0-20 \%$ ) et en fonction des paramètres physico-chimiques (moyenne et écart-type). Le coefficient moyen d'Abondance-Dominance est indiqué entre parenthèses.

\section{Table II}

Phytosociological table based on frequence of occurrence of species in community of Alsace Plain (V : 80-100\%, IV : 60-80\%, III : 40-60\%, II : 20-40\%, I : 0-20\%) and physico-chemical variables correlated (Means and standard deviation calculated on one year).

\begin{tabular}{|c|c|c|c|c|c|c|}
\hline 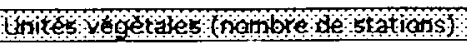 & $\mathrm{A}(9)$ & $\mathrm{Q}(\mathrm{X})$ & (1S) & 9012 & $E(20)$ & 260 \\
\hline Potamogeton coloratus & $v(2)$ & & & & & \\
\hline Batrachospermum monoliforme & $\|(1)$ & & & & & \\
\hline Juncus subnodulus & $\|(+)$ & & & & & \\
\hline Chara vulgaris & II (+) & & & & & \\
\hline Chara hispida & $\|(+)$ & & I(2) & & & \\
\hline Lamprocystis roseo persicina & $\|(+)$ & & $1(3)$ & I (t) & & \\
\hline Berula erecta & $\vee(2)$ & $\vee(4)$ & $v(2)$ & III (1) & III (1) & $I(+)$ \\
\hline Callitriche obtusangula & $1(+)$ & III $(+)$ & $\vee(2)$ & $v(2)$ & IV (1) & III (1) \\
\hline Lermna trisulca & & $1(1)$ & III (1) & III (1) & $\|(1)$ & \\
\hline Fontinalis antypyretica & & $1(+)$ & $1(1)$ & $\|(1)$ & $\|(+)$ & \\
\hline $\mathrm{Hode \textrm {mondmos }}$ & & & 10 & $81+1$ & (1) & $(1)$ \\
\hline Sparganium emersum & & & $\|(+)$ & il (1) & iI $(+)$ & iv (1) \\
\hline Lemna minor & & & II (1) & III (1) & $v(2)$ & $V(1)$ \\
\hline Potamogeton friseii & & & II (+) & $\|(1)$ & II (1) & $\|(1)$ \\
\hline 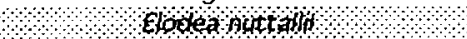 & & & dit & A(2) & $1(2)$ & $\sqrt{ }+1$ \\
\hline Ranunculus circinatus & & & $1(+)$ & & & \\
\hline Nasturtium officinale & & & & $V(1)$ & III $(+)$ & $\|(+)$ \\
\hline Spirodela polyrhiza & & & & $1(1)$ & II (1) & IV (1) \\
\hline Azolla filicoides & & & & I(1) & $1(1)$ & $\|(1)$ \\
\hline Groenlandia densa & & & & II (1) & $\|(1)$ & \\
\hline Potamogeton crispus & & & & III (1) & $\|(1)$ & $1(2)$ \\
\hline Myriophyllum verticillatum & & & & I(1) & $I(+)$ & \\
\hline Zannichellia palustris & & & & It (1) & $\|(1)$ & \\
\hline Hottonia palustris & & & & $1(1)$ & & \\
\hline Hippuris vulgaris & & & & I(1) & & \\
\hline Potamogeton pectinatus & & & & II (1) & IV (3) & IV (1) \\
\hline Myriophyllum spicatum & & & & II (1) & IV (1) & $v(1)$ \\
\hline Potamogeton perfoliatus & & & & $I(+)$ & $1(2)$ & III (1) \\
\hline Ceratophyllum demersum & & & & $I(+)$ & $V(2)$ & $V(1)$ \\
\hline Oenanthe fluviatilis & & & & & $1(+)$ & \\
\hline Ranunculus trichophyllus & & & & & $1(+)$ & \\
\hline Potamogeton pusillus & & & & & $1(t)$ & \\
\hline Ranunculus fluitans & & & & & II (2) & III $(+)$ \\
\hline Potamogeton lucens & & & & & & III (1) \\
\hline Potamogeton nodosus & & & & & & III (2) \\
\hline Mentha aquatica & $k(t)$ & $1(+)$ & 1(1) & $1(+)$ & $1(1)$ & \\
\hline Veronica anagallis & $1(+)$ & & $1(+)$ & $1(+)$ & $\|(+)$ & \\
\hline Myosotis scorpioides & $1(+)$ & & $1(1)$ & $H(+)$ & $\|(+)$ & \\
\hline Veronica beccabunga & & & & $1(+)$ & $1(+)$ & $1(+)$ \\
\hline $\mathrm{pH}$ & $7,4(0,1)$ & $7,5(0,2)$ & $7,5(0,1)$ & $7,6(0,2)$ & $7,9(0,2)$ & $7,9(0,2)$ \\
\hline Conductivité $(\mu \mathrm{S} / \mathrm{cm})$ & $608(115)$ & $736(112)$ & $740(99)$ & $657(66)$ & $657(63)$ & $508(52)$ \\
\hline Dureté (méq/l) & $4,8(1,4)$ & $4,7(0,9)$ & $5,0(0,7)$ & $3,9(0,4)$ & $3,8(0,5)$ & $3,2(0,3)$ \\
\hline $\mathrm{N}-\mathrm{NH}_{4}{ }^{+}(\mu \mathrm{g} / 1)$ & $14(7)$ & $22(14)$ & $45(28)$ & $34(31)$ & $61(40)$ & $255(107)$ \\
\hline $\mathrm{P}-\mathrm{PO}_{4}^{4} 3^{\circ}(\mu \mathrm{g} / \mathrm{I})$ & $7(2)$ & $13(6)$ & $15(7)$ & $29(24)$ & $40(33)$ & $192(116)$ \\
\hline $\mathrm{N}-\mathrm{NO}_{3}^{-}(\mathrm{mg} / \mathrm{l})$ & $5,5(1,4)$ & $5,3(1,8)$ & $4,7(2,1)$ & $2,9(2,5)$ & $1,6(1,1)$ & $2,5(0,9)$ \\
\hline
\end{tabular}


L'optimum de développement d'Elodea canadensis est atteint dans les groupements végétaux " $C$ " et “ $D$ " de l'échelle de bioindication de l'eutrophisation en milieu carbonaté calcaire. Cette espèce est en voie de régression dans les groupements " $E$ " et " $F$ ". En revanche, Elodea nuttallii se rencontre préférentiellement dans les groupements " $E$ " et " $F$ » (Tableau II).

D'une manière générale, les deux espèces cohabitent dans les eaux mésotrophes à eutrophes de la plaine d'Alsace, mais Elodea canadensis semble actuellement repoussée vers les secteurs mésotrophes (association " $C$ " et " $D$ ") ; les secteurs eutrophes (associations " $E$ " et " $F$ ") étant préférentiellement colonisés par $E$. nuttallii.

\section{Ecologie d'Elodea canadensis et d'Elodea nuttallii dans les cours d'eau des secteurs d'études}

\section{Tableau III}

Principales caractéristiques physico-chimiques des cours d'eau étudiés. Les valeurs correspondent à des valeurs stationnelles. Les minima et maxima mesurés pour chaque paramètre sont indiqués.

Table III

Characteristics of physico-chemical parameters analyzed in studied streams. Minima and maxima are expressed.

\begin{tabular}{|c|c|c|c|c|}
\hline & \multicolumn{2}{|c|}{ vos gos of Aord } & \multicolumn{2}{|c|}{ 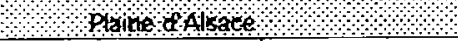 } \\
\hline & E.canadensis ( 8$)$ & E.nuttallii (6) & E.canadensis (15) & Enuttallii (20) \\
\hline Conductivité en $\mu \mathrm{S} / \mathrm{cm}$ & $65-90$ & $60-110$ & $660-740$ & $510-740$ \\
\hline $\mathrm{pH}$ & $6,8-7,2$ & $6,9-7,2$ & $7,5-7,9$ & $7,5-7,9$ \\
\hline $\mathrm{N}-\mathrm{NH}_{4}{ }^{+}$en $\mu \mathrm{g} / \mathrm{I}$ & $75-150$ & $50-330$ & $45-60$ & $45-255$ \\
\hline 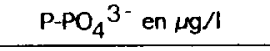 & $40-65$ & $40-200$ & $15-40$ & $15-190$ \\
\hline $\mathrm{N}-\mathrm{NO}_{3}$ en $\mathrm{mg} / \mathrm{l}$ & $0,3-1,0$ & $0,4-1,1$ & $1,6-4,7$ & $2,5-4,7$ \\
\hline
\end{tabular}

\section{Rôle des paramètres morphométriques}

Aucune influence significative de la profondeur, de la teneur en argiles et en sables n'a pu être mise en évidence pour expliquer la répartition des espèces d'élodées en fonction de paramètres " non trophiques" (ROBACH et al., 1996).

Elodea canadensis se rencontre dans les eaux courantes ou stagnantes, peu profondes, claires et relativement fraîches. Elodea nuttallii est observée dans des eaux calmes, à une profondeur allant de quelques centimètres à trois mètres. E. nuttallii semble avoir une amplitude écologique plus restreinte qu'Elodea canadensis vis-à-vis de la température et préférer les températures plus élevées (WOLFF, 1980).

\section{Rôle de la minéralisation}

La minéralisation influence les sensibilités spécifiques des macrophytes submergées et semble déterminer prioritairement la composition des phytocénoses aquatiques. Elodea canadensis et $E$. nuttallii ont été observées dans des eaux faiblement minéralisées, proches de la neutralité et peu chargées en azote nitrique des Vosges du Nord, alors qu'elles se développent dans les eaux minéralisées, neutres à légèrement alcalines et relativement chargées en azote nitrique de la plaine d'Alsace (figure 1). Ainsi, les deux espèces Elodea canadensis et $E$. nuttallii se sont installées, maintenues et développées dans les ruisseaux à minéralisation très différente dans le nord-est de la France. 



Figure 1

Distribution en classe de fréquence des deux espèces d'élodées pour les 4 paramètres physicochimiques $\mathrm{P}_{-} \mathrm{PO}_{4}{ }^{3-}, \mathrm{N}-\mathrm{NH}_{4}{ }^{+}, \mathrm{N}-\mathrm{NO}_{3}{ }^{-}$, conductivité.

Nota : Les surfaces représentées sont proportionnelles aux fréquences relatives $(\%$ du nombre de stations contenant l'espèce dont la moyenne annuelle du paramètre considéré est comprise dans la classe).

Figure 1

Distribution of the two elodeids according to 4 classes of phosphate, ammonia, nitrate concentrations and conductivity values. The distribution is expressed in $\%$ of occurrence frequency. 


\section{Rôle de la trophie}

Elodea canadensis préfère les cours d'eau mésotrophes ou modérément eutrophes, même si sa présence en plaine d'Alsace a été relevée dans des tronçons eutrophisés contaminés par des rejets de stations d'épuration, où elle côtoie Elodea nuttallii (figure 1). Bien qu'étant en régression, Elodea canadensis se maintient dans les secteurs mésotrophes. Dans notre zone d'étude, Elodea nuttallii présente un vaste spectre trophique, allant des cours d'eau mésotrophes jusqu'aux rivières hypertrophes (figure 1). Mais elle se développe préférentiellement dans les zones eutrophes, où elle concurrence fortement en plaine d'Alsace Elodea canadensis.

Ainsi, l'extension des deux espèces introduites s'explique par leur large amplitude écologique vis-à-vis de la trophie et de la minéralisation. Leur propagation est facilitée par leur mode de reproduction (dérive des rameaux, boutures, plantules...). Leur maintien et leur développement sont possibles car les exigences écologiques vis-à-vis de la température et du milieu physique (profondeur, vitesse du courant, substrat...) sont satisfaites.

\section{DISCUSSION : CONSÉQUENCES DE L'INTRODUCTION D'ELODEA CANADENSIS ET E. NUTTALLI/ DANS LES ÉCOSYSTĖMES AQUATIQUES}

Le mécanisme de l'apparition des hydrophytes introduits et de leur intégration au sein de phytocénoses en place a pu être défini chez Elodea canadensis. Différentes phases consécutives à son arrivée dans un hydrotope ont été distinguées (LEROY, 1976) :

- rupture d'équilibre avec phase d'expansion et occupation du maximum de place ;

- phénomènes de compétition et phase de régression pour des raisons d'ordre génétique, mais aussi d'autres liées au mode de reproduction asexuée (stolons) en Europe.

La phase actuelle est celle de l'intégration dans les phytocénoses aquatiques existantes.

Ces diverses étapes ne sont possibles que dans un milieu récepteur adapté aux exigences écologiques de ces macrophytes introduites (composition physico-chimique de l'eau, caractères mésologiques des cours d'eau).

\section{Compétition interspécifique}

MERIAUX et GEHU (1979) ont comparé, dans le Pas-de-Calais, des groupements végétaux dépourvus d'Elodea nuttallii et d'autres dans lesquels l'espèce introduite s'est implantée. Ils ont constaté que la compétition a surtout lieu avec Elodea canadensis et qu'une phase d'expansion d'Elodea nuttallii est en cours. D'autres travaux ont mis en évidence que l'extension d'Elodea nuttallii semble se faire au détriment d'E. canadensis (DUVIGNEAUD, 1976 ; LAMBINON et al., 1993). De plus, Elodea nuttallii peut constituer, à elle seule, la strate inférieure d'une communauté végétale et s'opposer au développement des autres espèces. $\mathrm{Ce}$ pouvoir compétitif conduit dans un premier temps à une diminution de la diversité floristique de la station, et à terme peut aboutir à une perte de la diversité biologique du cours d'eau.

Ainsi, Elodea nuttallii s'est développée au détriment d'une espèce protégée au niveau régional, Myriophyllum alterniflorum, dans les Vosges du Nord. Dans ce secteur, Elodea canadensis et $E$. nuttallii n'ont été observées ensemble que sur deux stations. En 1993, quelques pieds d'Elodea nuttallii y étaient mélangés à des peuplements importants d'E. canadensis. Fin 1995, la situation s'est pratiquement inversée. L'essentiel de la compétition a donc lieu entre les deux espèces d'élodées et en faveur d'Elodea nuttallii. Ce phénomène a été également mis en évidence dans les cours d'eau de la plaine d'Alsace (ROBACH et al., 1995 ; ROLLAND, 1995).

L'expansion d'Elodea nuttallii au détriment d'Elodea canadensis en plaine d'Alsace et, dans une moindre mesure, dans les Vosges du Nord s'explique par une eutrophisation croissante des milieux aquatiques. Cette dernière est plus compétitive vis-à-vis de l'absorption des orthophosphates, mais aussi vis-à-vis de l'azote ammoniacal, qu'Elodea canadensis. En effet, 
Elodea canadensis est plus sensible en milieu bicarbonaté calcique à l'azote ammoniacal qu' $E$. nuttallii (ROLLAND, 1995 ; ROLLAND et al., 1995). De plus, il a été démontré, en conditions de laboratoire, que le développement d'Elodea canadensis est optimal dans le cas d'une alimentation nitrique stricte alors que celui d'E. nuttallii est maximal dans le cadre d'un apport combiné d'azote ammoniacal et d'azote nitrique en proportions équimolaires et en proportions fortement majoritaires d'ions ammonium (ROLLAND et al., 1995). Elodea canadensis peut donc être considérée comme espèce " nitratophile ", alors qu'E. nuttallii serait " ammoniophile".

L'aptitude d'une espèce aquatique à stocker de grandes quantités de phosphore lui permettrait ainsi de résister aux importantes fluctuations temporelles de la charge phosphatée de l'eau, fluctuations caractéristiques des milieux eutrophes. Elodea nuttallii présente des capacités d'accumulation du phosphore plus importantes qu'E. canadensis, ce qui la rendrait plus compétitive en milieu eutrophe et pourrait, en partie, expliquer la distribution des deux espèces d'élodées (ROBACH et al., 1995, 1996).

\section{Epuration biologique}

La capacité d'épuration dépend du type de plante, de son taux de croissance, de la composition nutritive des eaux et de l'environnement physico-chimique du végétal. Le rôle des macrophytes dans la circulation des éléments chimiques minéraux dépend de l'accumulation par la biomasse vivante ou morte, et du relargage par la plante durant sa vie et au cours de sa décomposition (BLAKE, 1988).

Le caractère « ammoniophile " d'Elodea nuttallii lui confère la possibilité d'exercer un rôle épurateur des eaux, soit dans les systèmes lénitiques (OZIMEK et al., 1993), soit encore dans les systèmes lotiques, en vue d'épurer des eaux de station d'épuration ou des rejets de pisciculture (ROLLAND, 1995).

Le caractère " nitratophile " d'Elodea canadensis pourrait être utilisé à des fins de dépollution de sites atteints par une contamination nitratée chronique (ROLLAND, 1995).

Le rôle de " pompe à phosphates " joué par certains macrophytes est partiellement vérifié (BLAKE, 1988). En effet, Elodea nuttallii est capable, en conditions de laboratoire, de prélever du phosphore à partir du compartiment aqueux seul, ou dans les compartiments eausédiment (ROBACH et al., 1995). Le phénomène d'accumulation de phosphore dans les tissus végétaux est accentué lorsque la seule source de phosphore est la phase aqueuse (ROBACH et al., 1996). Des paramètres propres à l'espèce, anatomiques et physiologiques (structure de l'appareil végétatif, degré d'immersion, importance du système racinaire, mode de multiplication végétative...) influent sur la capacité des macrophytes à accumuler le phosphore (ROBACH et al., 1996). Les hydrophytes, présentant une large amplitude trophique et une bonne aptitude à se multiplier par fragmentation, se comportent en période estivale comme des agents de rétention temporaire du phosphore (ROBACH et al., 1996).

\section{Eutrophisation secondaire des eaux}

Le cycle biologique ne fait que déplacer le phosphore du compartiment eau vers le compartiment plante, en absence de faucardage et de récolte des plantes. Cela peut se traduire par une diminution temporaire de la trophie de l'eau, suite au stockage du phosphore dans les végétaux. Cependant, lors des premiers stades de la décomposition des plantes, une grande quantité de nutriments peut être libérée et contribuer ainsi à augmenter les apports en phosphore dans le milieu. Ce phénomène peut provoquer une eutrophisation du milieu, dont l'intensité dépend de la forme chimique sous laquelle sont évacués les nutriments (complexés à de la matière organique ou en tant que minéraux). Lorsque les hydrophytes ont stocké une forte quantité de nutriments dans leurs tissus et que ces derniers sont libérés sous forme minérale lors de la sénescence des macrophytes, le niveau trophique augmente et permet l'installation d'espèces eutrophes. Ceci expliquerait la présence des phytocénoses même dans des eaux dont les teneurs en phosphore et en azote ammoniacal sont temporairement faibles (exemple de la plaine d'Alsace du Steingriengiessen, du Schütz) où Elodea nuttallii est présente malgré des niveaux trophiques de l'eau faibles. 


\section{CONCLUSIONS ET PERSPECTIVES}

L'apparition d'Elodea nuttallii et Elodea canadensis dans le nord-est de la France a induit des modifications des communautés végétales au sein des écosystèmes aquatiques. Après des phases successives d'expansion des deux espèces, celles-ci ont développé des phénomènes de compétition entre elles et avec les autres espèces de la phytocénose aquatique. L'introduction d'Elodea canadensis et $E$. nuttallii peut donc conduire à un appauvrissement de la communauté végétale. Elodea canadensis révèle une sensibilité plus grande à l'azote ammoniacal qu'Elodea nuttallii, caractère expliquant en partie la répartition écologique des deux espèces. D'autre part, Elodea nuttallii qui paraît être l'espèce la plus compétitive, est la plus apte à accumuler le phosphore ; aptitude lui conférant une plus grande facilité à occuper les milieux eutrophes et la possibilité d'agir également sur la qualité des eaux des petits cours d'eau, dont les apports en nutriments demeurent modérés. Ces deux espèces pourraient alors être utilisées dans l'épuration des eaux, à la condition d'effectuer un faucardage. La récolte des plantes à la fin de la saison végétative n'est pas sans conséquences sur le fonctionnement des écosystèmes. Sinon, leur décomposition à la fin de la période de végétation, en relarguant des quantités importantes de nutriments, peut être la source d'une eutrophisation secondaire des eaux.

\section{BIBLIOGRAPHIE}

BLAKE G., 1988. Rôle des végétaux aquatiques vis-à-vis du phosphore dans les écosystèmes et les systèmes d'épuration in Le phosphore, ses dérivés et leur comportement dans le milieu naturel, Dijon, 22-24 novembre 1988, 1-14.

BRAUN-BLANQUET J., 1964. Pflanzensociologie. Springer Verlag, Wien, 1-865.

CARBIENER R., ORTSCHEIT A., 1987. Wasserpflanzengesellschaften als Hilfe zur Qualitätsüberwachung eines des grössten Grundwasservorkommen Europas (Oberrheinebene). Proceed. Intern. Symposium, Tokyo, 283-312.

CARBIENER R., TREMOLIERES M., MERCIER J.L., ORTSCHEIT A., 1990. Aquatic macrophyte communities as bioindicators of eutrophication in calcareous oligosaprobe stream waters (Upper Rhine plain, Alsace). Vegetatio, 86, 71-88.

DE LANGHE J.E., D'HOSE R., 1974. Prospections floristiques faites en 1973 en Argonne et en Lorraine. Natura Mosana, 26 (4), 117-120.

DUVIGNEAUD J., 1976. Le domaine universitaire de Sart Tilman et ses abords (Province de Liège, Belgique). Notes floristiques. Lejeunia N.S., 81, 1-63.

ENGEL R., MULLER S., WOLFF P., 1979. Contribution à la flore des Vosges du Nord. Bull. Soc. Hist. Nat. Moselle, 42, 105-111.

GEISSERT F., SIMON M., WOLFF P., 1985. Investigations floristiques et faunistiques dans le nord de l'Alsace et quelques secteurs limitrophes. Bull. Ass. Phil. Als. Lorr., 21, 111-127.

GODRON D.A., 1877. Arrivée à Nancy de l'Elodea canadensis. Bull. Soc. Sci. Nancy, série 2, 3, 99-102.

LAMBINON J., DE LANGHE J.E., DELVOSALLE L., DUVIGNEAUD J., VANDEN BERGHEN C., 1993. Nouvelle Flore de la Belgique, du Grand-Duché de Luxembourg, du nord de la France et des régions voisines (Ptéridophytes et Spermaphytes). Editions du Patrimoine du Jardin botanique national de Belgique, Meise, 4è édition, 1-1092.

LEROY R., 1976. Prolifération des végétaux aquatiques dans le cours de l'Erdre. Etude et moyens d'intervention. C.E.T.E., Equipement Nantes, 1-57.

MERIAUX J.L., 1977. Etude analytique et comparative de la végétation aquatique d'étangs et marais du nord de la France (Vallée de la Sensée et Bassin houiller du Nord/ Pas-de-Calais). Aspects physionomiques floristiques, systématiques, chorologiques et écologiques. Thèse Doct., Metz Doc. phytosoc. N.S.3, 1-244, Lille. 
MERIAUX J.L., 1979. Elodea nuttallii St. John, espèce nouvelle pour le nord de la France. Bull. Soc. Nat. France, $32(1-2), 30-32$.

MERIAUX J.L., GEHU J.M., 1979. Réactions des groupements aquatiques et subaquatiques aux changements de l'environnement. Epharmonie 1979, Tüxen, Cramer in der A.R. Gantner Verlag Kommanditgesellschaft, 121-142.

MULLER S., 1990. Une séquence de groupements végétaux bioindicateurs d'eutrophisation croissante des cours d'eau faiblement minéralisés des Basses Vosges gréseuses du nord. C. R. Acad. Sci. Paris, Série III, 310, 509-514.

OZIMEK T., VAN DONK E., GULATI R.D., 1993. Growth and nutrient uptake by two species of Elodea in experimental conditions and their role in nutrient accumulation in a macrophyte-dominated lake. Hydrobiologia, 251, 13-18.

ROBACH F., EGLIN I., CARBIENER R., 1991. Hydrosystème rhénan : évolution parallèle de la végétation aquatique et de la qualité de l'eau (Rhinau). Bull. Ecol., 22 (1), 227-241.

ROBACH F., MERLIN S., ROLLAND T., TREMOLIERES M., 1995. Approche écophysiologique de la bioindication de la qualité de l'eau par les plantes aquatiques : rôle du phosphore. In "Marqueurs biologiques de Pollution », 21-22 septembre 1995, Chinon, 171-187.

ROBACH F., MERLIN S., TREMOLIERES M., 1996. Relations entre la végétation aquatique et la qualité de l'eau en plaine d'Alsace. Rôle particulier du phosphore. Rapport Agence de l'eau Rhin-Meuse, 1-122.

ROLLAND T., 1995. Approche écophysiologique du rôle de l'azote ammoniacal dans la répartition de deux macrophytes aquatiques, Elodea canadensis Michx. et Elodea nuttallii St. John. Impact sur l'Activité Nitrate Réductase et l'Activité Photosynthétique. Thèse de Doct., Strasbourg, 1-303.

ROLLAND T., ROBACH F., TREMOLIERES M., DESTER S., 1995. L'Activité Nitrate Réductase chez les plantes aquatiques : un outil biologique du suivi de la contamination ammoniacale. In Marqueurs biologiques de Pollution, 21-22 septembre 1995, Chinon, 189-199.

SELL Y., 1959. Etude comparative de quelques espèces du genre Elodea à propos de l'apparition à Strasbourg et ses environs d'une espèce nouvelle pour l'Europe continentale. Bull. Ass. Phil. Als. Lorr., 10 (6), 121-133.

SELL Y., 1968. Les élodées. Aquarama, 2, 18-23.

SOCIÉTÉ D'ÉTUDE DE LA FLORE D'ALSACE, 1982. Flore d'Alsace (Plaine rhénane, Vosges, Sundgau), d'après ISSLER, LOYSON, WALTER (1952). Institut de Botanique, Strasbourg, 2è èd., 1-621.

THIEBAUT G., MULLER S., 1995. Nouvelles données relatives à la séquence de bioindication de l'eutrophisation dans les cours d'eau faiblement minéralisés des Vosges du Nord. Acta Botanica Gallica, 142 (6), 627-638.

WEBER-OLDECOP D.W., 1977. Elodea nuttallii St. John (Hydrocharitaceae), eine neue limnische Phanerogame der deutschen Flora. Arch. Hydrobiol., 79 (3), 397-403.

WOLFF P., 1980. Die Hydrilleae (Hydrocharitaceae) in Europa. Gött. Flor. Rundbr., 14 (2), 33-56. 